\title{
A COORDINATE-FREE APPROACH TO SURFACE KINEMATICS
}

\author{
by A. I. MURDOCH
}

(Received 15 April, 1989)

A thin three-dimensional material system, such as a thin shell or fluid-fluid interface, is often modelled as a bidimensional continuous body which at any instant "occupies" some geometrical surface. The time evolution of such surfaces is usually described in terms of curvilinear coordinates [2], [4], [6], a procedure which can mask the geometry involved. An alternative, coordinate-free, approach has been employed [1], [3] which patently exhibits the fundamental geometric (and algebraic) aspects of the kinematics of deforming surfaces. The foundations of this approach are presented in Section 2, following introductory remarks on notation and calculus in Euclidean point spaces, and hitherto unpublished results are developed in Section 3. Account is taken both of material and non-material surfaces: in the former case (surface) mass is conserved (this will be true for thin solid shells) while in the latter context mass exchange with contiguous phases is possible (as is to be expected in the case of fluid-fluid interfaces). The results are also pertinent to singular surfaces [2], [6], [7] (such as shock waves) which are not endowed with intrinsic material attributes but rather with discontinuities of bulk quantities.

1. Notation and preliminaries. Let $\mathscr{V}_{1}, \mathscr{V}_{2}$ denote finite-dimensional inner-product spaces over $\mathbb{R}$. Then $\operatorname{Lin}\left(\mathscr{V}_{1}, \mathscr{V}_{2}\right):=$ the space of all linear transformations from $\mathscr{V}_{1}$ into $\mathscr{V}_{2}$ and, when $\operatorname{dim} \mathscr{V}_{1}=\operatorname{dim} \mathscr{V}_{2}$,

$$
\operatorname{Invlin}\left(\mathscr{V}_{1}, \mathscr{V}_{2}\right):=\left\{\mathbf{A} \in \operatorname{Lin}\left(\mathscr{V}_{1}, \mathscr{V}_{2}\right): \mathbf{A} \text { invertible }\right\}
$$

When $\mathscr{V}_{2}=\mathscr{V}_{1}$ the foregoing sets are abbreviated to $\operatorname{Lin} \mathscr{V}_{1}$ and Invlin $\mathscr{V}_{1}$, respectively. The transpose $\mathbf{A}^{T}$ of $\mathbf{A} \in \operatorname{Lin}\left(\mathscr{V}_{1}, \mathscr{V}_{2}\right)$ is that element of $\operatorname{Lin}\left(\mathscr{V}_{2}, \mathscr{V}_{1}\right)$ defined by

$$
\mathbf{A}^{T} \mathbf{v}_{2} \cdot \mathbf{v}_{1}=\mathbf{v}_{2} \cdot \mathbf{A} \mathbf{v}_{1}
$$

for any $\mathbf{v}_{i} \in \mathscr{V}_{i}(i=1,2)$. Further,

$$
\begin{aligned}
& \operatorname{Orth}\left(\mathscr{V}_{1}, \mathscr{V}_{2}\right):=\left\{\mathbf{Q} \in \operatorname{Invlin}\left(\mathscr{V}_{1}, \mathscr{V}_{2}\right): \mathbf{Q}^{-1}=\mathbf{Q}^{T}\right\}, \\
& \operatorname{Orth} \mathscr{V}_{1}:=\operatorname{Orth}\left(\mathscr{V}_{1}, \mathscr{V}_{1}\right) \\
& \operatorname{Sym}^{+} \mathscr{V}_{1}:=\left\{\mathbf{S} \in \operatorname{Lin} \mathscr{V}_{1}: \mathbf{S}^{T}=\mathbf{S} \text { and } \mathbf{S} \text { positive-definite }\right\} \\
& \text { Sk } \mathscr{V}_{1}:=\left\{\mathbf{W} \in \operatorname{Lin} \mathscr{V}_{1}: \mathbf{S}^{T}=-\mathbf{S}\right\}
\end{aligned}
$$

The tensor product of $\mathbf{a}_{1}, \mathbf{b}_{1} \in \mathscr{V}_{1}$ is $\mathbf{a}_{1} \otimes \mathbf{b}_{1} \in \operatorname{Lin} \mathscr{V}_{1}$ defined by

It follows that

$$
\left(a_{1} \otimes b_{1}\right) \mathbf{v}_{1}:=\left(b_{1} \cdot v_{1}\right) a_{1} \text {. }
$$

$$
\left(a_{1} \otimes b_{1}\right)^{T}=b_{1} \otimes a_{1} \quad \text { and } \quad\left(a_{1} \otimes b_{1}\right)\left(c_{1} \otimes d_{1}\right)=\left(b_{1} \cdot c_{1}\right)\left(a_{1} \otimes d_{1}\right) .
$$

If $\mathbf{F} \in \operatorname{Invlin}\left(\mathscr{V}_{1}, \mathscr{V}_{2}\right)$ then the following polar decompositions hold:

$$
\mathbf{V R}=\mathbf{F}=\mathbf{R U} \text {, }
$$

Glasgow Math. J. 32 (1990) 299-307. 
where $\mathbf{V}, \mathbf{R}$ and $\mathbf{U}$ are unique, with $\mathbf{V} \in \operatorname{Sym}^{+} \mathscr{V}_{2}$,

$$
\mathbf{R} \in \operatorname{Orth}\left(\mathscr{V}_{1}, \mathscr{V}_{2}\right) \text { and } \mathbf{U} \in \operatorname{Sym}^{+} \mathscr{V}_{1}
$$

Intuitive notions concerning "space" are encapsulated and generalised in the concept of an Euclidean point space $\left(\mathscr{E}_{1}, \mathscr{V}_{1}\right)$. Elements of the set $\mathscr{E}_{1}$ are termed "points" and of the finite-dimensional inner-product space $\mathscr{V}_{1}$ over $\mathbb{R}$ "translations" or "displacements". The displacement necessary to reach point $\mathbf{y}$ from point $\mathbf{x}$ is denoted by $\mathbf{y}-\mathbf{x} \in \mathscr{V}_{1}$ and the point reached from $\mathbf{x}$ by undergoing a displacement $\mathbf{u}$ is denoted by $\mathbf{x}+\mathbf{u}$. Clearly, if $\mathrm{z} \in \mathscr{E}_{1}, \mathrm{v} \in \mathscr{V}_{1}$, then

$$
(\mathbf{z}-\mathbf{y})+(\mathbf{y}-\mathbf{x})=(\mathbf{z}-\mathbf{x}), \quad(\mathbf{x}+\mathbf{u})+\mathbf{v}=\mathbf{x}+(\mathbf{u}+\mathbf{v}), \quad \mathbf{x}+\mathbf{0}=\mathbf{x}, \quad \text { and } \quad \mathbf{x}-\mathbf{x}=\mathbf{0} .
$$

The foregoing may be formalised, but this is not necessary for our purposes. It is important only to notice that addition of "points" is meaningless and that no point is distinguished (as "origin"). In particular, if $\operatorname{dim} \mathscr{V}_{1}=1,2$ or 3 then $\left(\mathscr{E}_{1}, \mathscr{V}_{1}\right)$ is a model for a line, plane or "physical space", respectively. In what follows, $(\mathscr{E}, \mathscr{V})$ denotes "physical space". Any finite-dimensional inner-product space over $\mathbb{R}$ may be regarded as an Euclidean point space, "coinciding" with its space of translations. The space $\left(\mathscr{E}_{1}, \mathscr{V}_{1}\right)$ has the natural metric induced by the norm on $\mathscr{V}_{1}$ : thus $|\mathbf{y}-\mathbf{x}|$ defines the distance between points $\mathbf{x}$ and $\mathbf{y}$ where $|\mathbf{v}|:=(\mathbf{v} \cdot \mathbf{v})^{1 / 2}$.

If $f: \mathscr{D} \subset\left(\mathscr{E}_{1}, \mathscr{V}_{1}\right) \rightarrow\left(\mathscr{E}_{2}, \mathscr{V}_{2}\right)$ maps an open subset $\mathscr{D}$ of point space $\left(\mathscr{E}_{1}, \mathscr{V}_{1}\right)$ into point space $\left(\mathscr{E}_{2}, \mathscr{V}_{2}\right)$, then $f$ is said to be differentiable at $\mathbf{x} \in \mathscr{D}$ if there exists an $\mathbf{L} \in \operatorname{Lin}\left(\mathscr{V}_{1}, \mathscr{V}_{2}\right)$ such that $\left(\mathbf{h} \in \mathscr{V}_{1}\right)$

$$
f(\mathbf{x}+\mathbf{h})=f(\mathbf{x})+\mathbf{L h}+o(\mathbf{h}) \quad \text { as } \quad \mathbf{h} \rightarrow \mathbf{0} .
$$

The map $\mathbf{L}$ is termed the derivative (or gradient) of $f$ at $\mathbf{x}$ and denoted by $\nabla f(\mathbf{x})$. If $\nabla f$ exists on $\mathscr{D}$, so that

$$
\nabla f: \mathscr{D} \rightarrow \operatorname{Lin}\left(\mathscr{V}_{1}, \mathscr{V}_{2}\right)
$$

then it, too, may be differentiable at $\mathbf{x}$. If so this derivative is denoted by $\nabla \nabla f(\mathbf{x})$ and is an element of $\operatorname{Lin}\left(\mathscr{V}_{1}, \operatorname{Lin}\left(\mathscr{V}_{1}, \mathscr{V}_{2}\right)\right)$ and so identifiable as a bilinear mapping from $\mathscr{V}_{1} \times \mathscr{V}_{1}$ into $\mathscr{V}_{2}$. Such a mapping $\nabla \nabla f(\mathbf{x})$ is symmetric: that is

for all $\mathbf{u}, \mathbf{v} \in \mathscr{V}_{1}$.

$$
(\nabla \nabla f(\mathbf{x}) \mathbf{u}) \mathbf{v}=(\nabla \nabla f(\mathbf{x}) \mathbf{v}) \mathbf{u},
$$

2. Surfaces. $\dagger$ Intuitively, at each point $\mathbf{x}$ of a smooth surface $S$ in three-dimensional Euclidean space $\mathscr{E}$ there is a unique plane, $T_{\mathbf{x}}$ say, which most closely approximates $S$ near $\mathbf{x}$. This is the notion of the tangent plane to $S$ at $\mathbf{x}$. The collection $\mathscr{T}_{\mathbf{x}}$ of all displacements in $T_{\mathbf{x}}$ is a bidimensional subspace of the three-dimensional space $\mathscr{V}$ of all displacements in $\mathscr{E}$ and is termed the tangent space to $S$ at $\mathbf{x}$. Further, there would appear to be (at least near $\mathbf{x})$ a bijective correspondence between points $\mathbf{y}$ on $S$ and the feet $\mathbf{p}_{\mathbf{x}}(\mathbf{y})$ of the perpendiculars from these points onto $T_{\mathbf{x}}$. The foregoing may be formalised: a surface $S$ in $\mathscr{E}$ is a subset of $\mathscr{E}$ endowed with local structure at each $\mathrm{x} \in S$ by a bidimensional subspace $\mathscr{T}_{\mathbf{x}}$ of $\mathscr{V}$ together with a locally-unique map $\pi_{\mathbf{x}}$ such that the following conditions hold.

$\dagger$ The approach used here, specifically axioms S.1-3, is due to Walter Noll. 

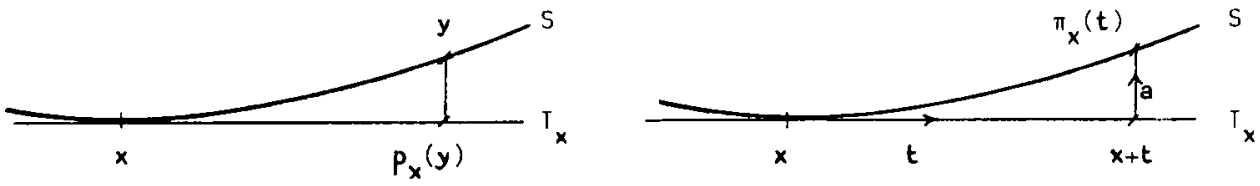

Figure 1

S.1. $\pi_{\mathrm{x}}: \mathscr{T}_{\mathrm{x}}^{0} \rightarrow \mathscr{E}$ with $\operatorname{Rg}\left(\pi_{\mathrm{x}}\right) \subset S$, where $\mathscr{T}_{\mathrm{x}}^{0}$ is an open neighbourhood of the zero vector 0 in $\mathscr{T}_{x}$, and $\pi_{x}$ is of class $C^{2}$ with the range of $\nabla \pi_{x}(t)$ (which maps $\mathscr{T}_{x}$ linearly into $\mathscr{V}$ ) bidimensional for each $\mathbf{t} \in \mathscr{T}_{\mathbf{x}}^{\mathbf{0}}$.

S.2. $\pi_{\mathbf{x}}(\mathbf{t})=\mathbf{x}+\mathbf{t}+\mathbf{a}$, where $\mathbf{a} \in \mathscr{T}_{\mathbf{x}}^{\prime}$ and is of order $o(\mathbf{t})$ as $\mathbf{t} \rightarrow \mathbf{0}$.

S.3. If $\mathbf{y} \in \operatorname{Rg}\left(\pi_{\mathrm{x}}\right)$ then $\pi_{\mathbf{y}}^{-1} \circ \pi_{\mathrm{x}}: \mathscr{T}_{\mathrm{x}}^{0} \rightarrow \mathscr{T}_{\mathbf{y}}^{0}$ is a $C^{2}$ diffeomorphism (possibly after suitably restricting the domains and codomains of $\pi_{x}, \pi_{y}$ ).

S.4. For some open neighbourhood $N_{\mathbf{x}}$ of $\mathbf{x}$ in $\mathscr{E}$ we have $\pi_{\mathbf{x}}\left(\mathscr{T}_{\mathbf{x}}^{0}\right) \cap N_{\mathbf{x}}=S \cap N_{\mathbf{x}}$.

Remarks. 1. Associated with $\pi_{\mathrm{x}}$ is the map $\hat{\pi}_{\mathrm{x}}: T_{\mathrm{x}}^{0} \rightarrow S$, where $T_{\mathrm{x}}^{0}:=\left\{\mathbf{y} \in \mathscr{E}: \mathbf{y}-\mathbf{x} \in \mathscr{T}_{\mathrm{x}}^{0}\right\}$ and, for each $\mathbf{t} \in \mathscr{T}_{\mathbf{x}}^{0}, \hat{\pi}_{\mathbf{x}}(\mathbf{x}+\mathbf{t})=\pi_{\mathbf{x}}(\mathbf{t})$. Clearly $\hat{\pi}_{\mathbf{x}}$ maps points in $T_{\mathbf{x}}$ near $\mathbf{x}$ into $S$ and is the inverse of $\mathbf{p}_{\mathbf{x}}$.

2. From S.2. $\pi_{x}$ is injective and, for any $y, z \in \operatorname{Rg}\left(\pi_{\mathbf{x}}\right)$, we have

$$
\pi_{\mathbf{x}}^{-1}(\mathbf{y})-\pi_{\mathbf{x}}^{-1}(\mathbf{z})=\mathbf{P}(\mathbf{x})(\mathbf{y}-\mathbf{z})
$$

where $\mathbf{P}(\mathbf{x}) \in \operatorname{Lin}\left(\mathscr{V}, \mathscr{T}_{\mathbf{x}}\right)$ denotes the perpendicular projection of $\mathscr{V}$ upon $\mathscr{T}_{\mathbf{x}}$.

3. Since S.2. implies $\pi_{\mathbf{x}}(0)=x$, so that

$$
\pi_{\mathbf{x}}(\mathbf{t})=\pi_{\mathbf{x}}(\mathbf{0})+\mathbf{t}+o(\mathbf{t}) \text { as } \mathbf{t} \rightarrow \mathbf{0}
$$

it follows that

$$
\nabla \pi_{\mathbf{x}}(\mathbf{0})=\mathbf{I}(\mathbf{x}),
$$

where $\mathbf{I}(\mathbf{x}) \in \operatorname{Lin}\left(\mathscr{T}_{\mathbf{x}}, \mathscr{V}\right)$ denotes the inclusion of $\mathscr{T}_{\mathbf{x}}$ in $\mathscr{V}$. That is, if $\mathbf{t} \in \mathscr{T}_{\mathbf{x}}$ then

$$
\mathbf{I}(\mathbf{x}) \mathbf{t}=\mathbf{t} .
$$

This map is to be distinguished both from the identity $1(x)$ on $\mathscr{T}_{\mathbf{x}}$ and the identity 1 on $\mathscr{V}$. Indeed, since if $t \in \mathscr{T}_{x}$ and $v \in \mathscr{V}$ then

$$
\mathbf{t} \cdot(\mathbf{l}(\mathbf{x}))^{T} \mathbf{v}=\mathbf{l}(\mathbf{x}) \mathbf{t} \cdot \mathbf{v}=\mathbf{t} \cdot \mathbf{v}=\mathbf{t} . \mathbf{P}(\mathbf{x}) \mathbf{v}
$$

it follows that

$$
\mathbf{I}(\mathbf{x})^{T}=\mathbf{P}(\mathbf{x})
$$

Of course,

$$
\mathbf{P}(\mathbf{x}) \mathbf{l}(\mathbf{x})=\mathbf{1}(\mathbf{x}) \text {. }
$$

4. If $y \in \operatorname{Rg}\left(\pi_{x}\right)$ and the domains and co-domains of $\pi_{y}$ and $\pi_{x}$ are suitably restricted so that $\pi_{y}^{-1} \circ \pi_{x}$ is a bijection from $\mathscr{T}_{x}^{0}$ onto $\mathscr{T}_{y}^{0}$, then S.3 implies that for every $t \in \mathscr{T}_{\mathbf{x}}^{0}$

$$
\nabla\left(\pi_{\mathbf{y}}^{-1} \circ \pi_{\mathbf{x}}\right)(\mathbf{t}) \in \operatorname{Invlin}\left(\mathscr{T}_{\mathbf{x}}, \mathscr{T}_{\mathbf{y}}\right)
$$

5. S.4 ensures that the topology induced on $S$ by its embedding in $\mathscr{E}$ coincides with the weakest topology on $S$ for which all maps $\pi_{x}$ are homeomorphisms. Thus at each of its 
points $S$ is locally homeomorphic to an open subset of a plane (its tangent plane at that point).

If $f: S \rightarrow \mathscr{C}^{\prime}$ denotes a map defined on $S$ which takes values in an Euclidean point space $\mathscr{E}^{\prime}$ (in particular, $\mathscr{E}^{\prime}$ could be $\mathbb{R}, \mathscr{E}$ or $\mathscr{V}$ ) then its surface derivative $\nabla_{S} f$ is defined by

$$
\nabla_{s} f(\mathbf{x}):=\nabla\left(f \circ \pi_{\mathbf{x}}\right)(\mathbf{0}) .
$$

Clearly $\nabla_{s} f(\mathbf{x}) \in \operatorname{Lin}\left(\mathscr{T}_{\mathbf{x}}, \mathscr{V}^{\prime}\right)$, where $\mathscr{V}^{\prime}$ denotes the space of translations between points of $\mathscr{E}^{\prime}$. If $\mathbf{t} \in \mathscr{T}_{\mathbf{x}}$ is a unit vector then $\left(\nabla_{S} f(\mathbf{x})\right) \mathbf{t}$ delivers the rate of change of $f$ at $\mathbf{x}$ along any smooth curve on $S$ to which $\mathbf{t}$ is tangent at $\mathbf{x}$. Further, if $f$ is defined spatially and is of class $C^{1}$ at $\mathbf{x}$ then [3, Proposition 3.3] $\nabla_{S} f(\mathbf{x})$ is the restriction to $\mathscr{T}_{\mathbf{x}}$ of $\nabla f(\mathbf{x}) \in \operatorname{Lin}\left(\mathscr{V}, \mathscr{V}^{\prime}\right)$.

With $\nabla \nabla \pi_{x}(0)$, a bilinear mapping from $\mathscr{T}_{\mathbf{x}} \times \mathscr{T}_{\mathbf{x}}$ into $\mathscr{V}$, and either of the two possible choices of unit normal fields $n$, can be identified a symmetric linear transformation $\mathbf{L}_{\mathbf{x}}(\mathbf{x})$ on $\mathscr{T}_{\mathbf{x}}$ via (here $\boldsymbol{\tau}, \mathbf{v} \in \mathscr{T}_{\mathbf{x}}$ are arbitrary)

$$
\mathbf{L}_{\mathbf{n}}(\mathbf{x}) \boldsymbol{\tau} \cdot \boldsymbol{v}:=\left(\nabla \nabla \pi_{\mathbf{x}}(\mathbf{0}) \tau\right) \mathbf{v} \cdot \mathbf{n}(\mathbf{x}) .
$$

It follows [3, Lemma 3.2] that $L_{n}$, the curvature tensor at $\mathbf{x}$ associated with orientation $\mathbf{n}$, satisfies

$$
\mathbf{I L}_{\mathbf{n}}=-\nabla_{s} \mathbf{n}
$$

or, equivalently,

$$
\mathbf{L}_{\mathbf{n}}=-\mathbf{P} \nabla_{s} \mathbf{n} \text {. }
$$

If $\mathbf{u}$ is a class $C^{1}$ vector field defined on $S$ and $\Sigma$ a subset of $S$, with piece-wise smooth boundary, which lies in the range of some local map $\pi_{\mathbf{x}}$, then

$$
\int_{\partial \Sigma} \mathbf{u} \cdot \mathbf{v}=\int_{\Sigma}\left\{\operatorname{div}_{S} \mathbf{u}+2 \kappa_{\mathbf{n}} \mathbf{u} \cdot \mathbf{n}\right\} .
$$

Here $v$ denotes the outward unit normal to $\partial \Sigma$ (a tangential vector at each point),

$$
\kappa_{\mathbf{n}}:=\frac{1}{2} \operatorname{tr} \mathbf{L}_{\mathbf{n}}
$$

the mean curvature, and

$$
\operatorname{div}_{s} \mathbf{u}:=\operatorname{tr}\left(\mathbf{P} \nabla_{s} \mathbf{u}\right)
$$

the surface divergence of $\mathbf{u}$. In (2.14) the trace operation tr is taken (at $\mathbf{x})$ in $\mathscr{T}_{\mathbf{x}}$. Result (2.12) is the surface divergence theorem, a particularly simple result in the case of tangential vector fields (for which $\mathbf{u}, \mathbf{n}=0$ ). The generalisation to class $C^{1}$ tensor fields $\mathbf{T}$ for which $\mathbf{T}(\mathbf{x}) \in \operatorname{Lin}\left(\mathscr{T}_{\mathbf{x}}, \mathscr{V}\right)$ is

$$
\int_{\partial \Sigma} \mathbf{T} \mathbf{v}=\int_{\Sigma} \operatorname{div}_{S} \mathbf{T}
$$

where for any $\mathbf{k} \in \mathscr{V}$ we have

$$
\left(\operatorname{div}_{S} \mathbf{T}\right) \cdot \mathbf{k}:=\operatorname{div}_{S}\left(\mathbf{T}^{T} \mathbf{k}\right) .
$$


3. Kinematics of a surface. Let $f: \hat{S} \rightarrow \mathscr{E}$, where $f(\hat{S})=S$ and $\hat{S}, S$ are surfaces. Suppose $f$ is $C^{2}$ regular (that is, for each $\hat{\mathbf{x}} \in \hat{S}$, the map $f \circ \hat{\pi}_{\hat{\mathbf{x}}}$ is of class $C^{2}$ with $\operatorname{Rg}\left(\nabla_{S} f(\hat{\mathbf{x}})\right)$ bidimensional) and has a $C^{2}$ inverse. If $f(\hat{\mathbf{x}})=\mathbf{x}$, then since $f \circ \hat{\pi}_{\hat{\mathbf{x}}}=\pi_{\mathbf{x}} \circ\left(\pi_{\mathbf{x}}^{-1} \circ f \circ \hat{\pi}_{\hat{\mathbf{x}}}\right)$, use of the chain rule and (2.1) yields

$$
\begin{aligned}
\nabla_{\hat{S}} f(\hat{\mathbf{x}}): & =\nabla\left(f \circ \hat{\pi}_{\hat{\mathbf{x}}}\right)(\mathbf{0}) \\
& =\left(\nabla \pi_{\mathbf{x}}(\mathbf{0})\right)\left(\nabla\left(\pi_{\mathbf{x}}^{-1} \circ f \circ \hat{\pi}_{\hat{\mathbf{x}}}\right)(\mathbf{0})\right) \\
& =\mathbf{I}(\mathbf{x}) \mathbf{P}(\mathbf{x}) \nabla_{\hat{S}} f(\hat{\mathbf{x}}) .
\end{aligned}
$$

It follows that $\mathscr{T}_{\mathbf{x}}=\operatorname{Rg}\left(\nabla_{\hat{S}} f(\hat{\mathbf{x}})\right)$. We write

$$
\overline{\mathbf{F}}(\hat{\mathbf{x}}):=\nabla_{\hat{S}} f(\hat{\mathbf{x}}), \quad \mathbf{F}(\hat{\mathbf{x}}):=\nabla\left(\pi_{\mathbf{x}}^{-1} \circ f \circ \hat{\pi}_{\hat{\mathbf{x}}}\right)(\mathbf{0}),
$$

and term $\mathbf{F}$ the deformation gradient corresponding to the deformation $f$. From (3.1)

$$
\begin{aligned}
& \mathbf{F}(\hat{\mathbf{x}}) \in \operatorname{Invlin}\left(\mathscr{T}_{\hat{\mathbf{x}}}, \mathscr{T}_{\mathbf{x}}\right), \\
& \overline{\mathbf{F}}(\hat{\mathbf{x}})=\mathbf{I}(\mathbf{x}) \mathbf{F}(\hat{\mathbf{x}}) \quad \text { and } \quad \mathbf{F}(\hat{\mathbf{x}})=\mathbf{P}(\mathbf{x}) \nabla_{\hat{S}} f(\hat{\mathbf{x}}) .
\end{aligned}
$$

The polar decomposition theorem (1.3) yields

$$
\mathbf{R U}=\mathbf{F}(\hat{\mathbf{x}})=\mathbf{V R},
$$

where $\mathbf{R} \in \operatorname{Orth}\left(\hat{\mathscr{T}}_{\hat{\mathbf{x}}}, \mathscr{T}_{\mathbf{x}}\right), \mathbf{U} \in \operatorname{Sym}^{+} \hat{\mathscr{T}}_{\hat{\mathbf{x}}}$ and $\mathbf{V} \in \operatorname{Sym}^{+} \mathscr{T}_{\mathbf{x}}$. The Jacobian of $f$ at $\hat{\mathbf{x}}$, which expresses the local area magnification factor associated with deformation $f$, is

From $(3.2)_{1},(3.3)_{2},(3.4)_{1}$ and (3.5),

$$
J_{f}(\hat{\mathbf{x}})=\operatorname{det} \mathbf{U}
$$

$$
J_{f}(\hat{\mathbf{x}})=\left\{\operatorname{det}\left[\left(\nabla_{\hat{S}} f(\hat{\mathbf{x}})\right)^{T}\left(\nabla_{\hat{S}} f(\hat{\mathbf{x}})\right)\right]\right\}^{1 / 2} .
$$

A motion for a surface $\hat{S}$ is a family of deformations of $\hat{S}$ parametrised by time: that is, with any instant $t$ in some time interval is associated a $C^{2}$ regular map $f(., t): \hat{S} \rightarrow \mathscr{E}$ with $S_{t}:=f(\hat{S}, t)$ a surface. The corresponding velocity field $\mathbf{u}(., t)$ on $S_{t}$ is defined by $\ddagger$

$$
\mathbf{u}(\mathbf{x}, t):=\stackrel{\circ}{f}(\hat{\mathbf{x}}, t), \quad \text { where } \quad \mathbf{x}:=f(\hat{\mathbf{x}}, t) .
$$

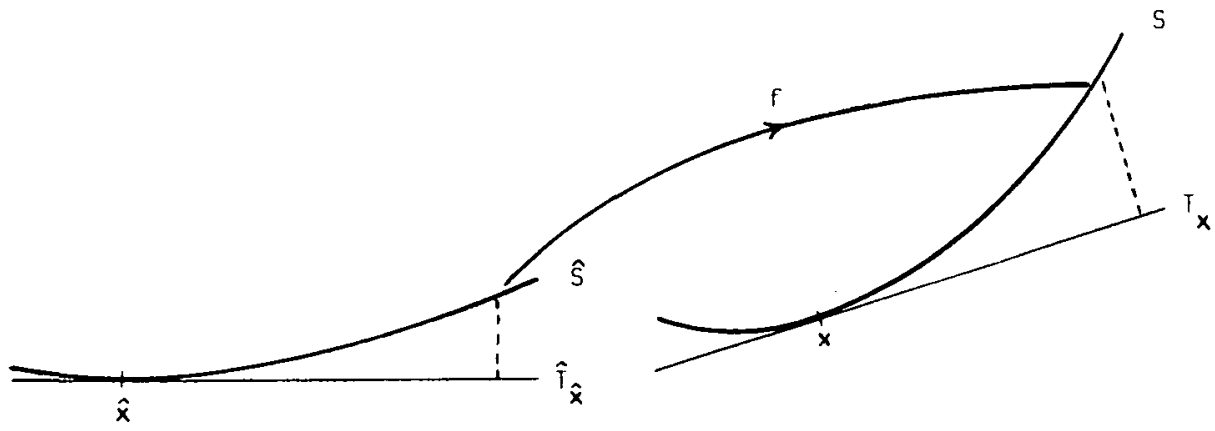

Figure 2

$\ddagger$ Here, and in what follows, a superposed circle will denote differentiation with respect to time holding fixed a point of $\hat{S}$. Thus in (3.9) below $\mathbf{n}(\mathbf{x}, t)$ represents $d / d t(\mathbf{n}(f(\hat{\mathbf{x}}, t), t))$, where $\mathbf{x}, \hat{\mathbf{x}}$ are related as in $(3.7)_{2}$. 

result

Aspects of the time evolution of surface geometry are contained in the following

Proposition.

$$
\nabla_{S} \mathbf{u}=\stackrel{2}{\mathbf{F}} \mathbf{F}^{-1}
$$

and

$$
\underline{\mathbf{n}}=-\left(\nabla_{S} \mathbf{u}\right)^{T} \mathbf{n}
$$

where, at instant $t, S:=S_{t}$.

Proof. If $\hat{\pi}_{\hat{\mathbf{x}}}\left(\pi_{\mathbf{x}}\right)$ denotes the local map defining $\hat{S}\left(S_{t}\right)$ near $\hat{\mathbf{x}}(\mathbf{x})$ then (3.7) may be expressed as a map on $\hat{\mathscr{T}}_{\hat{\mathbf{x}}}^{0}$ (the domain of $\hat{\pi}_{\hat{\mathbf{x}}}$ ); viz.

$$
\left(\mathbf{u}(., t) \circ \pi_{\mathbf{x}}\right) \circ\left(\pi_{\mathbf{x}}^{-1} \circ f(., t) \circ \hat{\pi}_{\hat{\mathbf{x}}}\right)=\stackrel{\circ}{f}\left(\hat{\pi}_{\hat{\mathbf{x}}}(.), t\right) .
$$

Differentiating this relation using the chain rule and evaluating at $\mathbf{0}$ yields (cf. (3.2) $)_{2}$, with $S:=S_{t}$,

$$
\nabla_{S} \mathbf{u}(\mathbf{x}) \mathbf{F}(\hat{\mathbf{x}}, t)=\nabla_{\hat{S}} \stackrel{\circ}{f}(\hat{\mathbf{x}}, t) .
$$

On assuming the order of differentiation may be interchanged (for this it suffices, as usual, that either mixed second derivative exist and be continuous),

$$
\nabla_{\hat{S}} \stackrel{\circ}{f}(\hat{\mathbf{x}}, t)=\stackrel{\circ}{\mathbf{F}}(\hat{\mathbf{x}}, t),
$$

(cf. (3.2) 1 ) whence, on suppressing arguments,

$$
\nabla_{S} \mathbf{u}=\stackrel{f}{\mathbf{F}} \mathbf{F}^{-1} \text {. }
$$

From $(3.3)_{1}, \mathbf{F}(\hat{\mathbf{x}}, t) v \in \mathscr{T}_{\mathbf{x}}$ for all $v \in \hat{\mathscr{T}}_{\hat{\mathbf{x}}}$. Thus

$$
\overline{\mathbf{F}}(\hat{\mathbf{x}}, t) \mathbf{v} \cdot \mathbf{n}(\mathbf{x}, t)=\mathbf{0}
$$

so that on $S_{t}$

$$
\overline{\mathbf{F}}^{T}(\hat{\mathbf{x}}, t) \mathbf{n}(\mathbf{x}, t)=\mathbf{0} .
$$

Differentiating (3.14) with respect to time, keeping $\hat{\mathbf{x}}$ fixed,

$$
\stackrel{8}{\mathbf{F}^{T}} \mathbf{n}+\overline{\mathbf{F}}^{T} \hat{\mathbf{n}}=\mathbf{0} .
$$

Hence, writing $\mathbf{F}^{-T}$ for $\left(F^{-1}\right)^{T}\left(=\left(F^{T}\right)^{-1}\right)$,

$$
\mathbf{F}^{-T} \stackrel{\mathfrak{F}}{T}^{T} \mathbf{n}=-\mathbf{F}^{-T} \overline{\mathbf{F}}_{\mathbf{i}} \mathbf{i}=-\mathbf{F}^{-T} \mathbf{F}^{T} \mathbf{P} \mathbf{n}=-\mathbf{P} \mathbf{n},
$$

on using (3.3) $)_{2}$ and (2.5). However,

$$
\mathbf{F}^{-T} \overline{\mathbf{F}}^{T}=\mathbf{F}^{-T}(\stackrel{\mathrm{F}}{\mathbf{F}})^{T}=\left(\stackrel{\varrho}{\mathbf{F}} \mathbf{F}^{-1}\right)^{T}=\left(\nabla_{S} \mathbf{u}\right)^{T}
$$

Further, since $\mathbf{n} \cdot \mathbf{n}=1$ it follows that

$$
\text { n̊. } \mathbf{n}=\mathbf{0}
$$

so that $\mathbf{n}$ is a tangential vector field and consequently

$$
\mathbf{P} \mathbf{n}=\mathbf{n} \text {. }
$$


From (3.15), (3.16) and (3.17)

$$
\stackrel{\mathbf{n}}{=}=-\left(\nabla_{S} \mathbf{u}\right)^{T} \mathbf{n} .
$$

It proves useful and instructive to extend $\mathbf{F}(\hat{\mathbf{x}}, t)$ to Invlin $\mathscr{V}$ in a simple and natural way: define $\mathbf{F}(\hat{\mathbf{x}}, t) \in \operatorname{Lin} \mathscr{V}$ by

$$
\left.\mathbf{F}(\hat{\mathbf{x}}, t)\right|_{\hat{\mathfrak{F}}_{\mathbf{i}}}=\overline{\mathbf{F}}(\hat{\mathbf{x}}, t)=\mathbf{I}(\mathbf{x}, t) \mathbf{F}(\hat{\mathbf{x}}, t) \text { and } \mathbf{F}(\hat{\mathbf{x}}, t) \hat{\mathbf{n}}(\hat{\mathbf{x}})=\mathbf{n}(\mathbf{x}, t) \text {. }
$$

Clearly, restriction of $\mathbf{F}(\hat{\mathbf{x}}, t)$ to $\hat{\mathscr{T}}_{\hat{\mathbf{x}}}$ (with range as codomain) yields $\mathbf{F}(\hat{\mathbf{x}}, t)$ so that $\mathbf{F}(\hat{\mathbf{x}}, t)$ is an extension of $\mathbf{F}(\hat{\mathbf{x}}, t)$. Further we require that $\mathbf{F}$ take $\hat{\mathbf{n}}$ into $\mathbf{n}$ and be linear on $\mathscr{V}$. From (3.19) it follows that the extended deformation gradient

$$
\mathbf{F}(\hat{\mathbf{x}}, t)=\mathrm{I}(\mathbf{x}, t) \mathbf{F}(\hat{\mathbf{x}}, t) \hat{\mathbf{P}}(\hat{\mathbf{x}})+\mathbf{n}(\mathbf{x}, t) \otimes \hat{\mathbf{n}}(\hat{\mathbf{x}}) .
$$

Here $\hat{\mathbf{P}}(\hat{\mathbf{x}})$ denotes the perpendicular projection of $\mathscr{V}$ upon $\mathscr{T}_{\hat{\mathbf{x}}}$. Suppressing arguments for brevity,

$$
\mathbf{F}=\mathbf{I F} \hat{\mathbf{P}}+\mathbf{n} \otimes \hat{\mathbf{n}} .
$$

Immediately this yields (recall (2.5))

$$
\mathbf{F}^{T}=\hat{\imath} \mathbf{F}^{T} \mathbf{P}+\hat{\mathbf{n}} \otimes \mathbf{n},
$$

where $\hat{\imath}(\hat{\mathbf{x}})$ denotes the inclusion of $\mathscr{\mathscr { T }}_{\hat{\mathbf{x}}}$ in $\mathscr{V}$. Less obviously,

$$
\mathbf{F}^{-1}=\hat{i} \mathbf{F}^{-1} \mathbf{P}+\hat{\mathbf{n}} \otimes \mathbf{n}=: \mathbf{G} \text {, say. }
$$

To verify (3.24) it is necessary only to compute FG, GF, and observe that

$$
\mathbf{I P}+\mathbf{n} \otimes \mathbf{n}=\mathbf{1}=\hat{\mathbf{l}} \hat{\mathbf{P}}+\hat{\mathbf{n}} \otimes \hat{\mathbf{n}} \text {. }
$$

Recalling the polar decomposition (3.4), the polar decompositions of $\mathbf{F}$ are

$$
\mathbf{R U}=\mathbf{F}=\mathbf{V R} \text {, }
$$

where

$$
\mathbf{U}=\hat{\mathbf{I}} \hat{\mathbf{P}}+\hat{\mathbf{n}} \otimes \hat{\mathbf{n}}, \quad \mathbf{V}=\mathbf{I V P}+\mathbf{n} \otimes \mathbf{n} \quad \text { and } \quad \mathbf{R}=\mathbf{I R} \hat{\mathbf{P}}+\mathbf{n} \otimes \hat{\mathbf{n}} .
$$

Of course, here $\mathbf{U}, \mathbf{V} \in \operatorname{Sym}^{+} \mathscr{V}$ and $\mathbf{R} \in$ Orth $\mathscr{V}$. The extended velocity gradient

$$
\mathbf{L}:=\text { Fे }^{-1} \text {. }
$$

Thus (cf. (3.20), (3.3) $2,(3.24)$ )

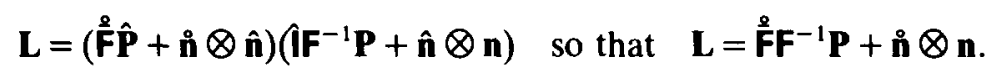

Clearly

$$
\stackrel{\mathbf{n}}{=} \mathbf{L n}
$$

and, from (3.8) and (3.9),

$$
\mathbf{L}=\left(\nabla_{S} \mathbf{u}\right) \mathbf{P}-\left(\nabla_{S} \mathbf{u}\right)^{T} \mathbf{n} \otimes \mathbf{n} .
$$

The stretching and spin tensors, $\mathbf{D}$ and $\mathbf{W}$, are defined by

$$
\mathbf{D}:=\frac{1}{2}\left(\mathbf{L}+\mathbf{L}^{T}\right), \quad \mathbf{W}:=\frac{1}{2}\left(\mathbf{L}-\mathbf{L}^{T}\right) .
$$


Upon writing $\overline{\mathbf{R}}:=\mathbf{I R}$ and noting

$$
\stackrel{2}{\mathbf{F}}=\stackrel{\check{\hat{\mathbf{R}}} \mathbf{U}}{=} \stackrel{\mathbf{R}}{\mathbf{U}}+\overline{\mathbf{R}} \mathbf{U} \text {, }
$$

(3.28) yields

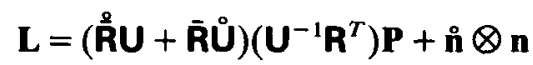

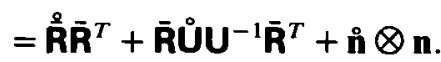

However, since $\mathbf{R}$ takes values in Orth $\mathscr{V}$, it follows that $\mathbf{R}^{T}$ takes values in $\mathrm{Sk} \mathscr{V}$. From $(3.26)_{3}$ this implies that

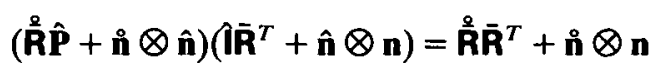

takes values in Sk $\mathscr{V}$. Hence (3.30) 1,2 and (3.31) yield

$$
\begin{aligned}
& \mathbf{D}=\frac{1}{2} \overline{\mathbf{R}}\left(\mathbf{U} \mathbf{U}^{-1}+\mathbf{U}^{-1} \mathbf{U}\right) \overline{\mathbf{R}}^{T}, \\
& \mathbf{W}=\frac{1}{2} \overline{\mathbf{R}}\left(\mathbf{U}^{-1}-\mathbf{U}^{-1} \mathbf{U}\right) \overline{\mathbf{R}}^{T}+\mathbf{R}^{\mathbf{R}} \overline{\mathbf{R}}^{T}+\stackrel{\mathbf{n}}{\otimes \mathbf{n}} \text {. }
\end{aligned}
$$

At this point no physical interpretation has been made concerning the concept of motion: such interpretation depends upon context. In the event that surface mass is conserved the surface is described as material [1], [2] and the appropriate velocity field $\mathbf{v}$ is merely the bidimensional analogue of the velocity field associated with a bulk phase. For singular surfaces [2], [6], [7] the corresponding velocity field is always orthogonal to the surface and so expressible in the form $U \mathbf{n}$. In the case of fluid-fluid interfaces the velocity field $\mathbf{u}_{S}$ is more subtle: at any instant its tangential component is associated with tangential mass transport [5] while its normal component corresponds to its singular surface nature (usually bulk mass density is discontinuous at such an interface). Of course, once the velocity field $u$ on the trajectory of a surface is established the appropriate interpretation of motion is thereby mandated: $\hat{S}$ is taken to be an instantaneous ( $t_{0}$, say) configuration and the motion $f$ relative thereto defined, for each $\hat{\mathbf{x}} \in \hat{S}$, to be the solution of the initial-value problem (cf. (3.7))

$$
\frac{d}{d t}\{f(\hat{\mathbf{x}}, t)\}=\stackrel{\circ}{f}(\hat{\mathbf{x}}, t)=\mathbf{u}(f(\hat{\mathbf{x}}, t), t), \text { where } f\left(\hat{\mathbf{x}}, t_{0}\right)=\hat{\mathbf{x}}
$$

The derivation of local forms of balance for surface thermodynamics involves use of the surface divergence theorem (cf. \$2) together with the appropriate Transport Theorem:

$$
\frac{d}{d t}\left\{\int_{\Sigma_{t}} \phi(., t)\right\}=\int_{\Sigma_{t}}\left\{\dot{\phi}+\phi \operatorname{div}_{S} \mathbf{u}\right\}
$$

Here $\Sigma_{t}:=f(\hat{\Sigma}, t)$, where $\hat{\Sigma} \subset \hat{S}$ is a subsurface of $\hat{S}$ with piecewise smooth boundary, and $\phi$ is a function defined on the trajectory of $\hat{S}$ under the motion prescribed by $\mathbf{u}$.

Proof.

$$
\frac{d}{d t}\left\{\int_{\Sigma_{t}} \phi(., t)\right\}=\frac{d}{d t}\left\{\int_{\hat{\Sigma}} \phi(f(., t), t) J_{f}(., t)\right\}
$$


where $J_{f}$ denotes the Jacobian of $f(., t)$ (cf. $(3.5,6)$ ). The theorem follows on differentiating under the integral sign and noting that (cf. (3.5)), on omitting arguments for brevity,

$$
\frac{d}{d t}\left(J_{f}\right)=\operatorname{tr}\left(\dot{U}^{-1}\right) J_{f}
$$

and

$$
\operatorname{div}_{S} \mathbf{u}=\operatorname{tr}\left(\mathrm{UUU}^{-1}\right)
$$

The result (3.36) follows from (3.8), (3.3),$(3.4)_{1}$, and (2.14) on observing that $\overline{\mathbf{R}}^{T} \mathbf{R}$ takes skew values $(\overline{\mathbf{R}}:=\mathbf{I R})$ since $\overline{\mathbf{R}}^{T} \overline{\mathbf{R}}$ is the identity on the relevant tangent space to $\hat{S}$ and is accordingly constant in time. Finally, it is instructive to compare the results of different time derivatives of a given quantity, $\phi$ say, defined on the trajectory of a surface. If $\dot{\phi}$ denotes its time derivative with respect to the motion associated with velocity field $\mathbf{u}$, and $\delta \phi / \delta t$ its time derivative with respect to the motion corresponding to the singular surface velocity $U \mathbf{n}$ (note all possible velocity fields must share this normal component), then

$$
\stackrel{\circ}{\phi}=\delta \phi / \delta t+\left(\nabla_{s} \phi\right) \mathbf{P u} .
$$

The proof of this result is non-trivial and is omitted in the interests of brevity.

\section{REFERENCES}

1. M. E. Gurtin and A. I. Murdoch, A continuum theory of elastic material surfaces, Arch. Rational Mech. Anal. 57 (1975), 291-323.

2. W. Kosiński, Field singularities and wave analysis in continuum mechanics (Ellis Horwood, 1986).

3. A. I. Murdoch and H. Cohen, Symmetry considerations for material surfaces, Arch. Rational Mech. Anal. 72 (1979), 61-98.

4. P. M. Naghdi, The theory of shells and plates, in Handbuch der Physik, Vol. VIa/2, ed. C. Truesdell (Springer Verlag, 1972).

5. L. G. Napolitano, Thermodynamics and dynamics of surface phases, Acta Astronautica 6 (1979), 1093-1112.

6. T. Y. Thomas, Plastic flow and fracture in solids (Academic Press, 1961).

7. C. A. Truesdell and R. A. Toupin, The classical field theories, in Handbuch der Physik, Vol. III/1, ed. S. Flügge (Springer Verlag, 1960).

Department of MATHEMATICS

UNIVERSITY OF STRATHCLYDE

GLASGOW G1 1XH

SCOTLAND 